\section{(D) Check for updates}

Cite this: Analyst, 2021, 146, 2689

\title{
Simultaneous measurement of free and conjugated estrogens in surface water using capillary liquid chromatography tandem mass spectrometry $\dagger$
}

\author{
Fan Huang, (D) ${ }^{a}$ Kersti Karu ${ }^{b}$ and Luiza C. Campos (DD *a
}

Given detrimental impacts induced by estrogens at trace level, determination of them is significant but challenging due to their low content in environmental samples and inherent weak ionisation. A modified derivatisation-based methodology was applied for the first time to detect estrogen in free and conjugated forms including some isomers simultaneously using liquid chromatography tandem mass spectrometry $\left(\mathrm{LC}-\mathrm{MS}^{n}\right)$. Derivatisation reaction with previously used 1,2-dimethyl- $1 \mathrm{H}$-imidazole-5-sulphonyl chloride allowed significant increase of mass spectrometric signal of analytes and also provided distinctive fragmentation for their confirmation even in complicated matrix. Then satisfactory recovery $(>75 \%)$ for the majority of analytes was achieved following optimisation of solid phase extraction (SPE) factors. The linearity was validated over a wide concentration with the correlation coefficient around 0.995. The repeatability of this methodology was also confirmed via the intra-day and inter-day precision and was less than 11.73\%. Validation of method quantification limits (MQLs) for all chosen estrogens was conducted using $1000 \mathrm{~mL}$ surface water, ranging from 7.0 to $132.3 \mathrm{pg} \mathrm{L}^{-1}$. The established methodology was applied to profile presence of targeted estrogens in natural surface water samples. Out of the ten compounds of interest, three free estrogens (E1, E2, E3) and two sulphate estrogens (E1-3S and E2-3S) were found over their MQLs, being in the range of $0.05-0.32 \mathrm{ng} \mathrm{L}^{-1}$.

Received 4th December 2020 Accepted 3rd March 2021

DOI: $10.1039 /$ dOan02335c

rsc.li/analyst
As indicated in previous research, an increased amount of circulating estrogens in bodies are associated with the development of breast cancer. ${ }^{10-12}$ Evidence-based literature ${ }^{13,14}$ interpreted mechanisms of estrogens responsible for carcinogenicity: (1) in process of metabolism, there is a quinone group forming at phenolic carbon position of estrogen derivatives, which likely react with DNA and corresponding DNA adducts arise. This modification and transformation happened on DNA structures likely give rise to mutation and even initiation of cancer; (2) as the significant substances for maintaining growth and development, estrogens and their metabolites exhibit functions of boosting mitogen and inhibiting apoptosis for cells, contributing to the promotion of cancer. Except for carcinogenicity, estrogens also result in intersexuality and disorder of reproductive capability. ${ }^{8}$

Excretion of estrogens from humans and animals is predominantly in the form of conjugates with glucuronide and sulphate salt, which are mostly biologically inactive as their poor hydrophobicity precluded the affinity between them and the estrogen receptors. ${ }^{15-17}$ In comparison, free estrogens including endogenous compounds estrone (E1), 17ß-estradiol (E2), and estriol (E3) and synthetically exogenous endocrine disruptors such as $17 \alpha$-ethinylestradiol (EE2), are less polar and more 
hydrophobic, rendering them more bioactive and potentially bioaccumulative. ${ }^{18}$ However, conjugated estrogens are subsequently readily hydrolysed to free forms by enzymes (e.g. arylsulfatase and glucuronidase) produced from microorganisms, bringing on potential higher risk after undergoing the reactivation process. ${ }^{19-21}$ Yet, measurement of estrogens, either free or conjugated forms, with accuracy and precision is raised according to its adverse clinical impact, especially at micro concentration level.

Detection techniques of estrogens developed continuously, such as assay test and mass spectrometry methods, are mostly used in recent years. ${ }^{22-24}$ Assays such as immunoassay and enzyme assay are usually capable of measuring estrogens with high sensitivity but behave susceptible to cross-reaction effects and variation among different antibodies, causing vulnerable accuracy of results. ${ }^{14,25-27}$ Furthermore, assay merely allows to quantify total estrogenic potency rather than exact content of each estrogens. In mass spectrometry methods, the use of gas chromatography-mass spectrometry (GC-MS) is restricted due to the non-volatile property of estrogens, limiting detection sensitivity and requiring large sample volumes. ${ }^{27}$ And for a real case, detection of estrogen metabolites with GC-MS is achieved by cleavage of their conjugated groups using glucuronidase and sulfatase firstly, followed by derivatisation process ${ }^{28}$ so quantification of free estrogens and estrogen conjugates simultaneously is complex and time-consuming. At present, liquid chromatography-mass spectrometry (LC-MS) is preferable for measuring estrogenic compounds among these methods. However, intrinsic weak ionisation of free estrogens limits their detection sensitivity, and complicated matrix constituents are likely to suppress signals of compounds. ${ }^{29}$ Thus, direct detection of estrogens with low concentrations can be only achieved with high-budget equipment, such as UPLC-MS/MS. ${ }^{30}$

As another alternative, derivatisation is the common pretreatment method which alters moieties of targeted compounds and enables their ionisation to intensify, augmenting signal response of under electrospray conditions using mass spectrometry (ESI-MS) instruments. To date, dansyl chloride is the most common derivatisation reagent to esterify estrogens E1, E2, E3 and EE2 for enhancing ionisation and increasing signal by ESI-MS. ${ }^{31-33}$ However, it was also noticed that dansyl labelled derivatives failed to offer distinctive confirmatory fragments in MS/MS due to nearly complete retention of charge at dansyl moiety, making this method problematic and susceptible to samples containing complex matrix. ${ }^{32}$ Furthermore, to our knowledge, existing methods were developed for only limited number of estrogens, which were almost free estrogens. ${ }^{12,31,34}$ In this study, we aimed to establish a sensitive analytical method for simultaneous detection of expansive estrogens including free and conjugate forms at a concentration level of sub-nanogram per litre, in which there are some structural isomers. Derivatives allowing diagnostic precursors and fragmentation would be desirable to increase accuracy of the method, especially in the presence of interferences. This could be achieved via derivatisation with 1,2-dimethylimidazole-5-sulfonyl chloride (DMIS), which has been applied to measure E2 in human serum prior to this study. ${ }^{35}$ Moreover, representative estrogenic glucuronide isomers were found to produce unique mass characteristics for further identification in the light of selective occurrence of derivatisation reaction. In addition to the capillary LC-MS ${ }^{n}$ method, we also optimised an offline solid phase extraction (SPE) extractions protocol. Following validation of procedures, this methodology was modified to profile, for the first time, four free estrogens and their main conjugated forms in natural water sampled from Regent's Park, London.

\section{Materials and methods}

\subsection{Chemicals, reagents and materials}

The standards of all selected free estrogens and their conjugates were obtained from the following suppliers. The analytical grade of four free estrogens E1, E2, E3, EE2 with purity higher than 98\% and estrone-3-sulfate sodium salt (E1-3S) were all purchased from Sigma-Aldrich (Dorset, UK). The remaining estrogen conjugates, estrone-3-glucuronide sodium salt (E1-3G), $17 \beta$-estradiol-3-sulfate sodium salt (E2-3S), $17 \beta$-estradiol-3-( $\beta$-D-glucuronide) sodium salt $(\mathrm{E} 2-3 \mathrm{G}), 17$ $\beta$-estradiol-17-( $\beta$-D-glucuronide) sodium salt (E2-17G), and estriol-3-sulfate sodium salt (E3-3S) were obtained from Santa Cruz biotechnology (California, US). The physiochemical properties and chemical structures of the above estrogens are presented in Table S1.†

Ammonium formate with purity over 99.0\%, ammonia hydroxide solution for analysis (28\%), formic acid (FA) with purity higher than $99.0 \%$ used for analysis, and reagent grade of sodium carbonate anhydrous and sodium bicarbonate, were all purchased from Fisher Scientific (Lancashire, UK). 1,2Dimethyl-1 $H$-imidazole-5-sulphonyl chloride (DMIS) was purchased from Apollo Scientific Ltd (Cheshire, UK). Sodium hydroxide $(\mathrm{NaOH})$ and sulphuric acid $\left(\mathrm{H}_{2} \mathrm{SO}_{4}\right)$ used to adjust $\mathrm{pH}$ value of water samples were acquired from Sigma-Aldrich (Dorset, UK).

All reagents, chromatographic grade methanol $(\mathrm{MeOH})$, acetonitrile, acetone, and ethyl acetate, used for SPE and LC-MS analysis were ordered from Fisher Scientific (Lancashire, UK). Milli-Q water (resistivity $18.2 \mathrm{M} \Omega \mathrm{cm}^{-1}$ ) applied in this study was produced from PURITE Select Analyst 320 system (CAT. L300275, UK).

For evaluating retention capability of SPE cartridges, polymeric reversed sorbent Oasis HLB (Waters, Hertfordshire, UK), Strata-X (Phenomenex, Cheshire, UK) and Supel ${ }^{\text {TM}}$-Select HLB (Sigma-Aldrich, Dorset, UK) were compared by the recovery of selected compounds, their characteristics were listed in Table 1.

\subsection{Stock and working standard solutions}

Individual standard stock solutions of estrogenic chemicals were prepared by dissolving $1 \mathrm{mg}$ of E1, E2, E3, EE2, E1-3G, 
Table 1 Characteristics of cartridges used to optimise recovery of estrogens

\begin{tabular}{lllll}
\hline Cartridges & Description & Sorbent particle size $(\mu \mathrm{m})$ & Sorbent weight $(\mathrm{mg})$ & Syringe barrel size $(\mathrm{mL})$ \\
\hline Oasis HLB & Polymeric reversed-phase sorbent & $30-60$ & 200 & 6 \\
Strata-X & Polymeric reversed-phase sorbent & $33-100$ & 200 & 6 \\
Supel ${ }^{\text {TM-Select HLB }}$ & $\begin{array}{l}\text { Hydrophilic modified styrene polymeric } \\
\text { reversed-phase sorbent for polar and }\end{array}$ & $50-70$ & 200 & 6 \\
& hydrophilic compounds & &
\end{tabular}

E2-3G and E3-3S, $2 \mathrm{mg}$ of E1-3S and E2-3S, $1.5 \mathrm{mg}$ E2-17G in $10 \mathrm{~mL}$ methanol with volumetric flasks, getting stock solutions at concentrations of $100-200 \mathrm{mg} \mathrm{L}^{-1}$. Then these stock solutions were stored in $-20{ }^{\circ} \mathrm{C}$ freezer. We applied the method stated in $\mathrm{Xu}$, Roman ${ }^{14}$ 's study, by measuring absolute peak area of each selected estrogen and estrogen conjugate with using capillary-LC-MS; all stock solutions were verified to be stable for at least 3 months under this temperature. Single working standard solutions of each estrogen with concentration of $1 \mathrm{mg} \mathrm{L}{ }^{-1}-2 \mathrm{mg} \mathrm{L}^{-1}$ were prepared by diluting the stock solutions with methanol. In addition, a mixed working standard solution of all targeted compounds at a concentration of $10 \mathrm{mg} \mathrm{L}^{-1}$ was prepared weekly by diluting aliquots of each estrogen stock solutions together in methanol, to attain concentration points for calibration curve.

\subsection{Sample extraction and derivatisation}

Milli-Q water spiked with multi-level concentrations of estrogen was used to develop solid phase extraction method. The recovery behaviour was investigated by extracting $10 \mathrm{~mL}$ spiked Milli-Q water at concentration of $1 \mu \mathrm{g} \mathrm{L}^{-1}$; the recovery value was defined as the ratio between the peak area of extracted samples derived from chromatographic analysis and that of standard solution having concentration of $10 \mu \mathrm{g} \mathrm{L} \mathrm{L}^{-1}$ after infusing into the instrument.

Prior to SPE pre-treatment, $\mathrm{pH}$ of water samples was adjusted to $\sim 7$ with $0.1 \mathrm{M} \mathrm{NaOH}$ or $\mathrm{H}_{2} \mathrm{SO}_{4}$ solution and then filtered through $0.45 \mu \mathrm{m}$ WCN cellulose acetate membrane (Whatman $^{\mathrm{TM}}$, Maidstone, UK). Cartridges hold by J.T. Baker ${ }^{\circledR}$ SPE-12G processor were conditioned with $6 \mathrm{~mL} \mathrm{MeOH}$ and $6 \mathrm{~mL}$ Milli-Q water first to activate sorbent. After loading the water sample using Automated Solid-Phase Extraction (Fisher Scientific, Lancashire, UK) at $5 \mathrm{~mL} \mathrm{~min}^{-1}$, and waiting the equilibrium between analytes and sorbents for 15 minutes, the SPE cartridge was rinsed with $8 \mathrm{~mL}$ washing solution for removing extra interferences and was dried with air vacuum pump for $1 \mathrm{~h}$. As reported by previous studies for monitoring estrogens, the cartridges after enriching 250-2000 mL natural water samples were well washed with 1-10 $\mathrm{mL}$ washing solution for removing impurities. ${ }^{36-42}$ The volume of washing solution applied in this study (i.e. $8 \mathrm{~mL}$ ) is within this usual volume range. Then the washing solutions with different composition, Milli-Q water, $5 \%(\mathrm{v} / \mathrm{v}) \mathrm{MeOH}$ solution and $10 \%(\mathrm{v} / \mathrm{v})$ $\mathrm{MeOH}$ solution with volume of $8 \mathrm{~mL}$ were further assayed.

Following elution by $8 \mathrm{~mL}$ of the selected solvent, the elute collected in glass conical tube was evaporated to dryness under a gentle nitrogen gas flow and the dry residue was reconstituted in $75 \mu \mathrm{L}$ sodium bicarbonate buffer $(50 \mathrm{mM}, \mathrm{pH}$ 10.5) and then $75 \mu \mathrm{L}$ of $1 \mathrm{mg} \mathrm{mL}{ }^{-1}$ DMIS dissolved in acetone (preparation procedure of DMIS is described in the ESI $\dagger$ ). After vortex for $2 \mathrm{~min}$, reaction vials involving solutions were heated at $60{ }^{\circ} \mathrm{C}$ for $15 \mathrm{~min}$ (optimisation of derivatisation method is presented in the ESI $\dagger$ ). The reaction mixture cooled down to ambient temperature was extracted with $2 \mathrm{~mL}$ of $n$-hexane for twice to remove excessive salt and interferences brought by the derivatisation procedure. Then the organic layer was transferred out and dried under nitrogen gas again, the dry residues were reconstituted in $1 \mathrm{~mL}$ of mobile phase (acetonitrile with $0.1 \%$ formic acid), the resulting mixed solution was stored in freezer at $-20{ }^{\circ} \mathrm{C}$ until further analysis. Prior to infusing into LC-MS, all samples were filtered through $0.22 \mu \mathrm{m}$ PTFE syringe filter (Gilson, Dunstable, UK).

\subsection{LC-MS optimisation}

The capillary LC-MS ${ }^{n}$ analysis was established for derivatisedand conjugated-estrogens using an Accela liquid chromatograph system (Thermo Fisher Scientific, Lancashire, UK) coupled to a Finnigan linear ion trap (LTQ) mass spectrometer. Ten $\mu \mathrm{L}$ of sample was injected through $20 \mu \mathrm{L}$-loop to a Hypersil GOLD $\mathrm{C}_{18}$ capillary column $(100 \mathrm{~mm} \times 2.1 \mathrm{~mm}$ i.d., particle size: $1.9 \mu \mathrm{m}$, Thermo Fisher Scientific, Lancashire, UK). Mobile phases were (A) water containing $0.1 \%$ formic acid and (B) acetonitrile containing $0.1 \%$ formic acid. The flow rate was $180 \mu \mathrm{L} \mathrm{min}{ }^{-1}$. Gradient programme was as follows: the ratios of solvent A and B were linearly changed to $20 \%$ and $80 \%$ respectively within $10 \mathrm{~min}$ from initial $70 \% \mathrm{~A}$ and $30 \% \mathrm{~B}$, then solvent B further went up to $90 \%$ in next $8 \mathrm{~min}$. Following a $7 \mathrm{~min}$ hold at $10 \% \mathrm{~A}$ and $90 \% \mathrm{~B}$, gradient program was run to an initial gradient condition within $1 \mathrm{~min}$ and maintained for $5 \mathrm{~min}$. The LC column was kept at the ambient temperature through the LC-MS analysis. The LC effluent was directed into an electrospray ionisation (ESI) source of a LTQ mass spectrometer. The mass spectrometer was operated in $\mathrm{MS}^{n}$ mode to monitor analytes. Nitrogen was used as ESI nebulision and as drying, whereas argon as a collision gas. The ESI parameters were set as: capillary temperature, $280{ }^{\circ} \mathrm{C}$; capillary voltage, $33 \mathrm{~V}$; ion source voltage, $4.5 \mathrm{kV}$; sheath gas flow, $40 \mathrm{~L} \mathrm{~min}^{-1}$; Aux gas flow, $10 \mathrm{~L} \mathrm{~min}{ }^{-1}$; and drying gas flow, $15 \mathrm{~L} \mathrm{~min}^{-1}$. The optimal tuning conditions were obtained by a direct infusion of individual working standard solution prepared at $1 \mathrm{mg} \mathrm{L}^{-1}$ with automatic syringe pump, and sample was injected into system at a flow rate of 
Table 2 Precursor ions and fragment ions of targeted unconjugated and conjugated estrogens under selected collision energy

\begin{tabular}{lllll}
\hline Estrogens & $\begin{array}{l}\text { Molecular } \\
\text { weight (Da) }\end{array}$ & $\begin{array}{l}\text { Precursor } \\
\text { ions } m / z\end{array}$ & $\begin{array}{l}\text { Collision } \\
\text { energy (\%) }\end{array}$ & $\begin{array}{l}\text { Representative MS }{ }^{2} \text { fragment ions, } m / z \\
\text { (\% relative abundance) }\end{array}$ \\
\hline E1-DMIS & 428 & 429 & 45 & $365(100), 161(33), 270(8), 251(6)$ \\
E2-DMIS & 430 & 431 & 45 & $367(100), 253(46), 161(27), 272(13)$ \\
E3-DMIS & 446 & 447 & 45 & $383(100), 251(35), 161(24), 269(11), 288(10)$ \\
EE2-DMIS & 454 & 455 & 45 & $391(100), 161(18), 277(7), 296(2)$ \\
E2-17G-DMIS & 606 & 607 & 35 & $431(100), 413(62), 253(4)$ \\
E1-3S & 350 & 349 & -35 & $269(100), 145(36), 159(30), 253(13)$ \\
E1-3G & 446 & 445 & -45 & $269(100), 113(52), 427(46)$ \\
E2-3S & 352 & 351 & -40 & $271(100), 145(35), 283(9)$ \\
E2-3G & 448 & 447 & -30 & $271(100), 429(93), 325(85), 113(20)$ \\
E3-3S & 368 & 367 & -35 & $287(100), 309(35), 80(21)$
\end{tabular}

$15 \mu \mathrm{L} \min ^{-1}$. The LTQ mass spectrometer was set at ESI full scan mode at $\mathrm{m} / \mathrm{z}$ of $90-750$ followed by MS/MS for each estrogen at collision energies $30-45 \%$, representative fragment ions from their dissociation patterns were recorded as summarised in Table 2.

LC-MS analyses were performed to establish the retention time and ESI mass spectra for each analytes. For the quantification of free estrogens-DMIS and E2-17G-DMIS derived from the derivatisation reaction, the LTQ mass spectrometer was set in positive (ESI + ) mode with an ESI full scan to measure precursor ions and then $\mathrm{MS}^{2}$ event of each precursor $[\mathrm{M}+\mathrm{H}]^{+}$ion with an isolation width of $2 \mathrm{~m} / \mathrm{z}$. In contrast, negative (ESI-) mode was employed for monitoring deprotonated molecular ion $[\mathrm{M}-\mathrm{H}]^{-}$of authentic conjugated estrogens at full scan mode, excluding E2-17G. Subsequently, events of MS $^{2}$ spectra on these five precursor ions were also created upon chosen collision energies. Thus, analysis of derivatised and native estrogens was done in two separate runs, during which the same LC method while opposite polarities for ESI ionisation were used.

Xcalibur software (Thermo Fisher Scientific) was used for data processing. Specifically, reconstructed ion chromatogram (RIC) was constructed for describing the intensity of targeted molecules with a given $\mathrm{m} / \mathrm{z}$ value.

\subsection{Method validation}

Method developed in this study was validated subject to the Food and Drug Administration (FDA) guidance ${ }^{43}$ by evaluating the linearity regression of analytes, limit of detection and quantification with LC-MS, inter- and intra-precision for analysis, selectivity, matrix effect, and finally recovery performance of established SPE method. Natural water collected from a lake of the Regent's Park was employed to conduct the validation tests.

Quantification of estrogens was proceeded by the standard addition method using matrix-matched calibration, which was prepared by adding aliquots of estrogen standard solution over a wide concentration spanning from individual instrument quantification limit (IQL) to $1000 \mu \mathrm{g} \mathrm{\textrm {L } ^ { - 1 }}$ into the extraction of $1000 \mathrm{~mL}$ natural surface water. Besides more economic cost, the standard addition method allows for measurement of analytes and is more tolerant to matrix effects in comparison with other quantitative method. ${ }^{44}$
The linearity was then evaluated over the chosen concentration points by the correlation coefficient value $\left(R^{2}\right)$ given by corresponding calibration curves.

According to the literature, the instrument detection limit (IDL) and IQL were experimentally estimated as the concentration of analytes when the instrument showed signal to noise $(\mathrm{S} / \mathrm{N})$ as 3 and 10 , respectively, ${ }^{45,46}$ which were established by measuring standards with gradually decreasing concentrations, until getting the closest values which yielded the matching $\mathrm{S} / \mathrm{N}$ ratios. Then the method detection limit (MDL) and method quantification limit (MQL) were identified using the surface water with spike of estrogens on a given enrichment factor, which were calculated following equation from ref. 47:

$$
\mathrm{MDL}=(\mathrm{IDL} \times 100) /\left(\mathrm{Re}_{\text {absolute }} \times \mathrm{EF}\right)
$$

where IDL refers to the instrument detection limit $\left(\mathrm{ng} \mathrm{L}^{-1}\right)$, $\mathrm{Re}_{\text {absolute }}$ is the absolute recovery of estrogens (\%) in natural surface water matrix, EF is the enrichment factor, specifically being 1000 herein. For the calculation of MQL, IQL was in placed of IDL in eqn (1).

For checking repeatability of this analytical method, intraday precision was obtained by calculating the relative standard deviation (RSD) of samples measured in 6 replicates $(n=6)$ and inter-day precision was assessed by conducting the interday procedure among four days $(n=4)$. Both assays used quality control samples (QC) with concentration at $0.1 \mu \mathrm{g} \mathrm{L}^{-1}$ for all chosen compounds. According to the guideline from FDA, selectivity was defined as the capability of an analytical method to quantify and verify the targeted analytes in the presence of interfering components from the matrix. ${ }^{48}$ The selectivity of our method was studied by the analysis of non-spiked natural water samples and estrogens-spiked sample at IQL level $(n=3)$. Significant signals from interference should not be detected at the corresponding retention time of the chosen compounds. $^{49}$

As the presence of matrix effects causes instability of accuracy and reproducibility to the established method, in particular when the ESI source was applied for analysis. To determine matrix effects arising from the surface water, the slope of matrix matched calibration curve was divided by the slope 
derived from the neat standard calibration curve, which is expressed as:

$$
\text { Matrix effect }(\%)=\left(1-S_{\text {matrix }} / S_{\text {neat }}\right) \times 100
$$

where $S_{\text {neat }}$ is the calibration curve slope of targeted compounds standards in neat mobile phase, $S_{\text {matrix }}$ is the calibration curve slope corresponding to the same standards spiked into the post-extraction of surface water.

The extraction of the method was validated by triplicate analysis of surface water $(1000 \mathrm{~mL})$ with spiked estrogens at three concentration levels of 50,100, and $1000 \mathrm{ng} \mathrm{\textrm {L } ^ { - 1 }}$. Nonspiked surface water with the same volume was also extracted in the meantime. The absolute and relative recovery percentage of estrogens with a known content were calculated based on the values quantified with the neat standard and matrix matched calibration by the following equations:

$$
\begin{aligned}
\operatorname{Re}_{\text {absolute }}(\%)= & \left(P_{\mathrm{SPE}}-P_{\text {control }}\right) /\left(P_{\text {neat standard }}\right) \times 100 \% \\
\operatorname{Re}_{\text {relative }}(\%)= & \left(P_{\mathrm{SPE}}-P_{\text {control }}\right) /\left(P_{\text {matrix standard }}-P_{\text {control }}\right) \\
& \times 100 \%
\end{aligned}
$$

where Re refers to the recovery of estrogens, $P_{\mathrm{SPE}}$ and $P_{\text {control }}$ are the peak areas of estrogens with a given spiked concentration in surface water and non-spiked control after the extraction procedure, $P_{\text {neat standard }}$ and $P_{\text {matrix standard }}$ are the peak areas of corresponding standard solutions in neat solvent and in the matrix post-extraction, respectively. It demonstrates the loss of analytes arising from the SPE procedure.

\section{Results and discussion}

\subsection{Mass spectra of derivatised estrogens}

There was observed poor ESI ionisation of individual E1, E2, E3 and EE2 standard solution prepared at $10 \mathrm{mg} \mathrm{L}^{-1}$ when analytes were directly infused or injected on the column, which is subsequently connected to the LTQ MS or the single quadrupole MS system. From the data processing window, neither strong ESI response nor clear chromatographic peaks were discovered. In previous work, it was concluded that sensitive response of MS was able to be achieved when the analytes were already ionised in solutions. ${ }^{50}$ As listed in Table S1, $\uparrow$ acid dissociation constant at logarithmic scale ( $\left.\mathrm{p} K_{\mathrm{a}}\right)$ of E1, E2, E3 and EE2 are 10.25, 10.27, 10.25 and 10.24, respectively, ${ }^{51}$ following the equations for calculating percent ionisation of acidic and basic chemicals: ${ }^{52}$

$$
\begin{aligned}
& \text { percent ionisation }(\%)=\frac{100}{1+10^{\left(\mathrm{p} K_{\mathrm{a}}-\mathrm{pH}\right)}} \\
& \text { percent ionisation }(\%)=\frac{100}{1+10^{\left(\mathrm{pH}-\mathrm{p} K_{\mathrm{a}}\right)}} .
\end{aligned}
$$

Results obtained indicate that ionisation of free estrogens in the range of acid to neutral $\mathrm{pH}$ solution is limited. The lack of ionisable groups in unconjugated estrogens, such as carboxy and amine, is the main reason leading to their negligible ionis- ation. ${ }^{53}$ Given less-polar properties for unconjugated estrogens, atmospheric pressure chemical ionisation (APCI) is theoretically more recommendable to obtain a higher signal compared with ESI mode ${ }^{54}$ while the improvement was found minimal in our trial. Therefore, derivatisation reagent DMIS was employed to incorporate cationic label into analytes for driving enhancement of detection sensitivity. Nitrogen atom in DMIS made derivatives more ionisable than their parent compounds, which was ascribed to the presence of two tertiary amines possessing easily protonated properties and the generation of conjugate-acid ammonium ion which owns a low $\mathrm{p} K_{\mathrm{a}}$ value. ${ }^{31,55}$ From ESI mass spectra and chromatographic results, it is noticeable that the derivatisation reaction by DMIS occurred with estrogens bearing phenolic hydroxyl group, e.g., four free estrogens and E2-17G, but inactive toward estrogen compounds merely possessing aliphatic hydroxyl group. The detailed reaction took place as shown in Fig. 1. This characteristic is consistent with Keski-Rahkonen's ${ }^{35}$ finding on the reaction between E2 and DMIS. The same selective reactions toward phenolic hydroxyl groups were also observed when using other proposed sulphonyl chloride derivatisation reagents such as dansyl chloride, pyrindine-3-sulphonyl chloride and 1,2-dimethylimidazole-4-sulphonyl chloride. ${ }^{56-58}$

In earlier studies, dansyl chloride was the most common derivatisation reagent for measuring estrogens and their metabolites. ${ }^{14,31,32,53}$ In all of these publications, it was observed that the fragment ion showing critical abundance was 5-(dimethylamino)-naphthalene moiety, which was presumed to solely from dansyl label dissociated from the parent ions in the light of molecular structure. ${ }^{31}$ Herein, it was not diagnostic adequately to monitor compound transition and susceptibility to interferences in complicated matrices. Likewise, a positional isomer of DMIS, 1,2-dimethylimidazole-4-sulfonyl chloride, also allowed the improvement of detection for E2 or other phenolic compounds by forming dimethylimidazole sulfonyl derivatives via a nucleophilic substitution reaction; however the fragment ions at $\mathrm{m} / \mathrm{z} 159, \mathrm{~m} / \mathrm{z} 144$ and $\mathrm{m} / \mathrm{z} 96$ found dominantly were almost entirely from the dimethylimidazole sulfonyl and dimethylimidazole moieties. ${ }^{58,59}$ Nevertheless, DMIS tagged estrogens in this study displayed more peculiar cleavage schemes by generating characteristic fragment ions containing estrogen moiety. The different dissociation schemes may originate from the relative positions of chargeable groups (e.g. amine and imine groups) in precursor ions.

The development of derivatisation procedure is described in detail within the ESI. $\uparrow$ As confirmed by the linear ion trap MS, DMIS derivatives produced stable protonated ions at $\mathrm{m} / \mathrm{z}$ $429,431,447,455,607$, namely $[\mathrm{M}+159]^{+}$under positive electrospray (Fig. 1). Under $\mathrm{MS}^{2}$ monitoring of each precursor ion, representative product ions subject to $\mathrm{MS} / \mathrm{MS}$ were identified and their relative abundance was described in Table 2. It was observed that derivatives of unconjugated estrogens exhibited analogous cleavage pathways activated by collision energy. Undergoing dissociation of precursor ion at $\mathrm{m} / \mathrm{z} 429$ for E1, charged fragment ions $m / z 365$ and $m / z 161$ dominated relying on their high relative abundance. The former is assigned as 


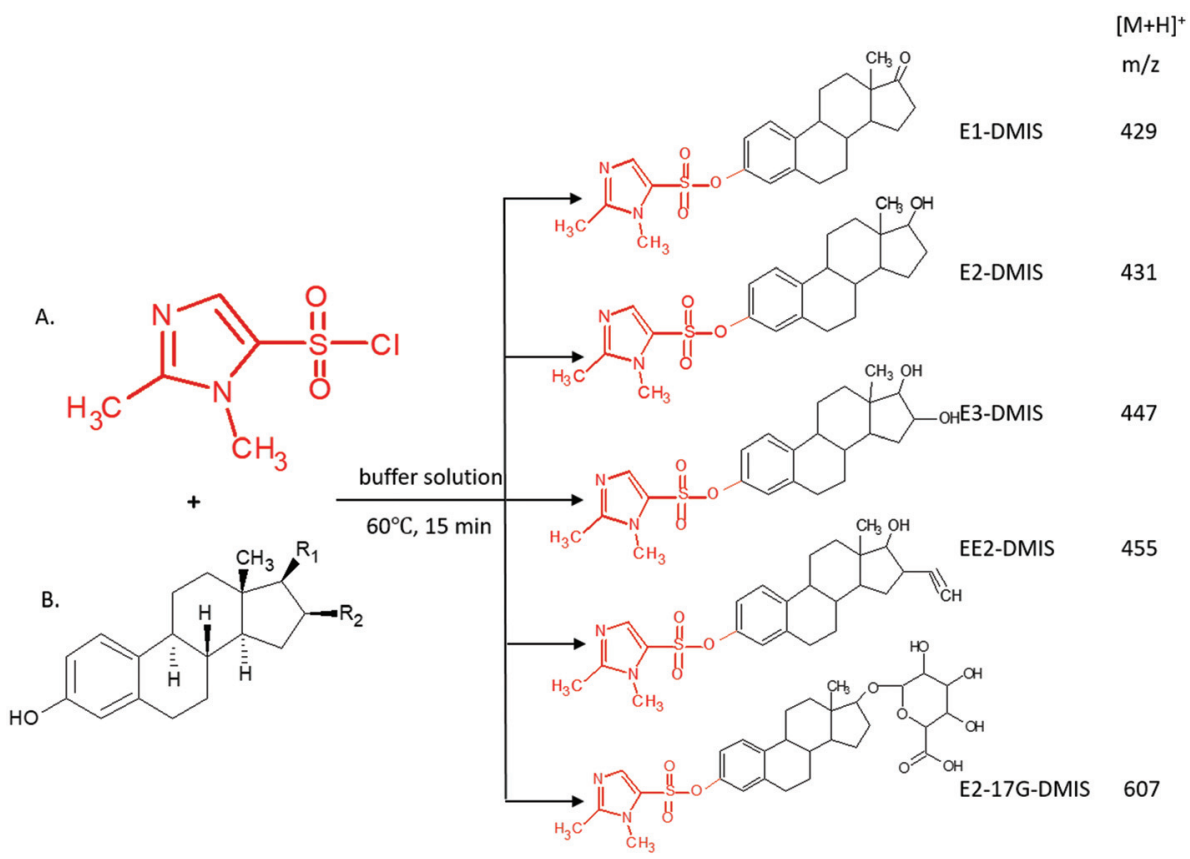

Fig. 1 Derivatisation reaction between selected estrogens containing phenolic hydroxyl and DMIS: (A) DMIS; (B) estrogen compounds involving C-3 hydroxyl groups, e.g., E1, E2, E3, EE2 and E2-17G.

$\left[\mathrm{C}_{23} \mathrm{H}_{31} \mathrm{~N}_{2} \mathrm{O}_{2}\right]^{+}$, arising from the loss of $\mathrm{SO}_{2}$ from $\mathrm{E} 1$ derivatives as $\left[\mathrm{C}_{23} \mathrm{H}_{31} \mathrm{~N}_{2} \mathrm{O}_{2}\right]^{+}$, arising from the loss of $\mathrm{SO}_{2}$ from E1 derivatives and rearrangement consequently. This resembling dissociation mechanism was also elucidated in previous study analysing DMIS, pyridine-3-sulfonyl chloride and dansyl chloride derivatives of estrogens. ${ }^{35,59} \mathrm{~A}$ fragment ion at $\mathrm{m} / \mathrm{z} 161$ corresponded to $\left[\mathrm{C}_{5} \mathrm{H}_{9} \mathrm{~N}_{2} \mathrm{O}_{2} \mathrm{~S}\right]^{+}$with structure of dimethylimidazole moiety affixing $\mathrm{SO}_{2}$, which has been stated before. $^{35,60}$ The dissociation ions at $\mathrm{m} / \mathrm{z} 270$ and $\mathrm{m} / \mathrm{z} 251$ corresponded to a radical E1 cation and a cyclic compound transformed from $\mathrm{E} 1$ ion after the loss of angular methyl and bond transition in ring moiety, which has a formula of $\left[\mathrm{C}_{17} \mathrm{H}_{15} \mathrm{O}_{2}\right]^{+}$. The less prominent fragmentations at $\mathrm{m} / \mathrm{z} 337$ and $\mathrm{m} / \mathrm{z} 309$ were designated as rearranged compounds, attaching dimethylimidazole moiety to dissociation intermediates of estrogen nucleus after ring cleavage. Similar cleavage schemes were discussed before. ${ }^{59,61}$ According to the informative spectra, primary suggested fragmentation schemes for E1-DMIS are proposed in Fig. 2.

For other free estrogens, E2, E3 and EE2, the dissociation patterns for their derivatives showed analogous to E1. Their mass spectra demonstrated an increment by $+2,+18$ and +26 to $\mathrm{m} / \mathrm{z}$ of E1 derivatives and its partial corresponding fragment ions. However, peaks at $\mathrm{m} / \mathrm{z} 429$ and $\mathrm{m} / \mathrm{z} 251$ were noticed under profiling E3-DMIS at $\mathrm{m} / \mathrm{z} 447$ upon collision activation. These two fragment ions were also observed in the positive fragmentation spectrum of E1 derivatives. We assumed that they originated from a loss of $\mathrm{H}_{2} \mathrm{O}$ from precursor ion $\mathrm{m} / \mathrm{z} 447$ and product ion $\mathrm{m} / \mathrm{z} 269$ of E3-DMIS respectively, undergoing an rearrangement analogous to pinacol reaction. An ion at $\mathrm{m} / \mathrm{z}$ 161 was found in fragmentations of all derivatised estrogens, making it possible to support that this product ion is indeed from labelling reagent. The dissociation pathways of the remaining derivatised estrogens were portrayed in Fig. S2 in the ESI. $\dagger$

The application of chemical derivatisation also enables differentiation between positional isomers of E2-3G and E2-17G by its selective reaction with the phenolic hydroxyl group. In their native forms, it is found that their full ESI mass spectra not only show their precursor ions $[\mathrm{M}-\mathrm{H}]^{-}$at $m / z 447$ but also the product ions $\mathrm{m} / \mathrm{z}$ at 271 with high abundance are entirely the same ${ }^{62,63}$ which makes it difficult to achieve identification of E2-3G and E2-17G especially in complicated matrix. Upon derivatisation with DMIS label, a positive ESI mass spectrum shows a peak at $m / z 607$ corresponding to E2-17G-DMIS. MS ${ }^{2}$ spectra on $\mathrm{m} / \mathrm{z} 607$ shows the fragments ions at $\mathrm{m} / \mathrm{z} 431$ and $\mathrm{m} / \mathrm{z}$ 413 for E2-17G-DMIS; the former was originated from the loss of monodehydrated glucuronic acid with $176 \mathrm{Da}$, which was also demonstrated for the derivatisation of propranolol metabolites; ${ }^{60}$ the latter was presumed to form after further dehydration at C-17 position. Less prominent fragment ion at $\mathrm{m} / \mathrm{z} 543$ corresponded to the loss of $\mathrm{SO}_{2}$ from E2-17G-DMIS as E1-DMIS did. Additionally, E2-containing specific ions at $\mathrm{m} / \mathrm{z} 367$ and $\mathrm{m} / \mathrm{z}$ 253 were both discovered as seen in $\mathrm{MS}^{2}$ spectrum of E2-DMIS.

Because the ionisation technique owned by the used LTQ instrument and the enhancement of signal intensities, the ESI-based method was applied in our study. Also, previous studies reported that ESI ionisation had a better performance in quantifying estrogens derivatised with dansyl chloride or 2-fluoro-1-methylpyridinium-ptoluenesulfonate (FMP-TS) in comparison with other ionisations such as APCI and atmospheric pressure photoionization (APPI) ${ }^{14,34,64}$ However, APPI 


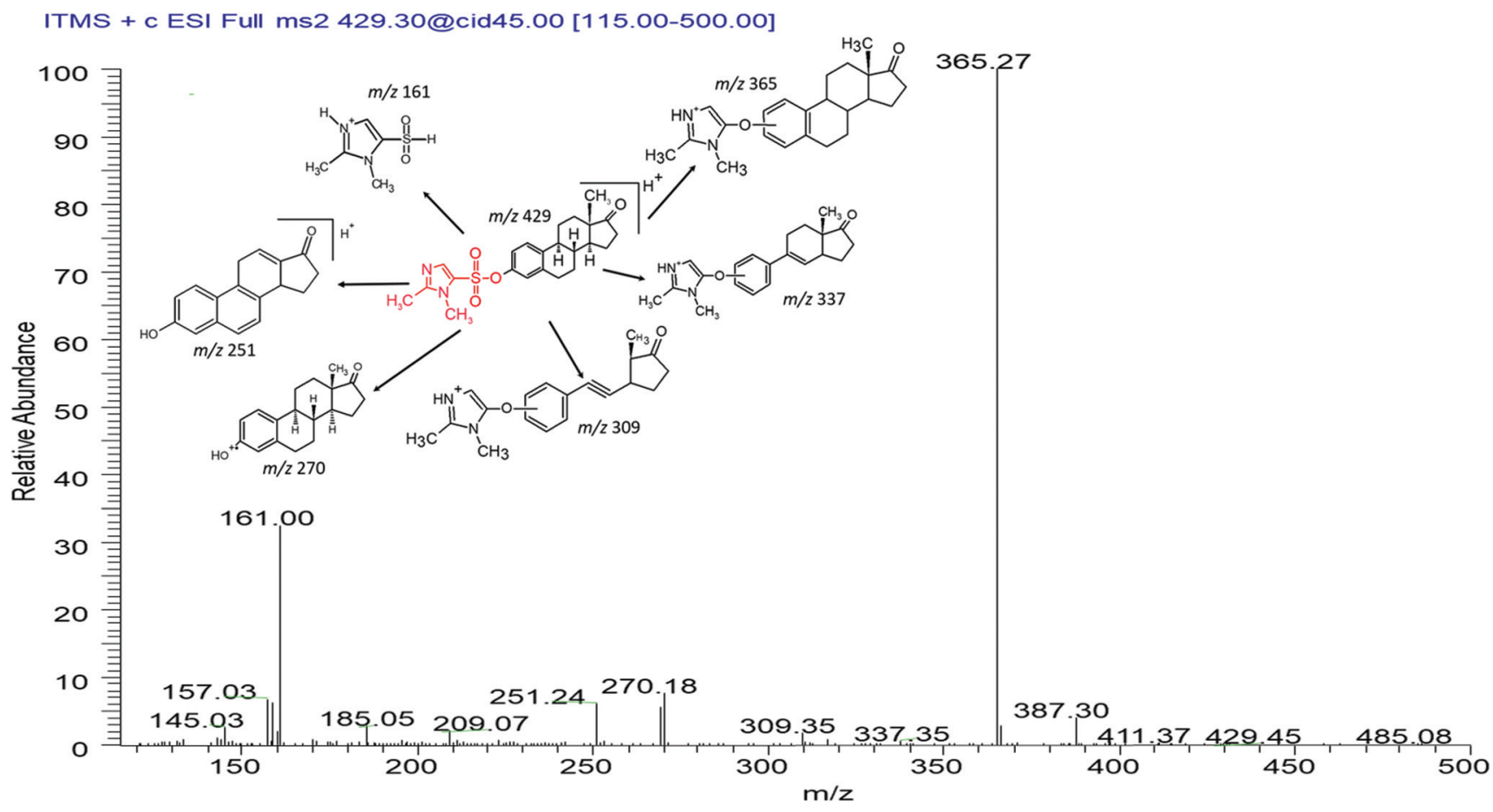

Fig. $2 \mathrm{MS}^{2}$ spectra of E1-DMIS acquired at the collision energy $45 \%$, and proposed dissociation scheme from its precursor ion at $m / z 429$.

was found preferable to ESI and APCI for measuring derivatised E2 in human serum using the DMIS as it is less prone to interferences of complicated matrix, although all three ionisation techniques achieved the same sensitivity using matrixfree standard. ${ }^{35}$ It was noticed that the ion suppression of ESI was not greater than APCI and APPI when the surface water matrix was analysed. ${ }^{64}$ Therefore, application of ESI ionisation was feasible in this study, while application of other soft ionisation methods could be further investigated for other complex matrices, such as sludge, food and tissue.

\subsection{Mass spectra of non-derivatised estrogen conjugates}

The deprotonated $[\mathrm{M}-\mathrm{H}]^{-}$ions were observed as precursor ions for the conjugated form of estrogens E1-3S, E1-3G, E2-3S, E2-3G and E3-3S. These precursor ions were subjected to $\mathrm{MS}^{2}$. $\mathrm{MS}^{2}$ spectra showed their unique fragment ions, which were investigated in order to identify these molecules of interest in samples containing complicated interferences. The first and most abundant fragment ions of both glucuronide and sulphate conjugates were their corresponding free estrogens, E1 $(\mathrm{m} / \mathrm{z}$ 269), E2 ( $\mathrm{m} / \mathrm{z} 271)$ and E3 $(\mathrm{m} / \mathrm{z} 287)$, which attributes to a loss of their sulphate/glucuronide group during MS/MS. Additionally, a fragment ion $\left[\mathrm{C}_{10} \mathrm{H}_{9} \mathrm{O}\right]^{-}$at $m / z 145$ was also present in sulphate estrogens except for E3-3S, the latter has a peak at $\mathrm{m} / \mathrm{z} 80$ with a presumed structure of $\left[\mathrm{SO}_{3}\right]^{-}$originated from fragment of sulphate moiety. For E1-3G and E2-3G, the glucuronide ring is brittle and yielded fragment $\left[\mathrm{C}_{5} \mathrm{H}_{5} \mathrm{O}_{3}\right]^{-}$ion at $\mathrm{m} / z$ 113. These dissociation patterns were aligned with observation in the previous studies. ${ }^{65-67}$ The typical fragment ions for all underivatised estrogens are summarised in Table 2.
A mixed solution containing all estrogens was tested to investigate the loss of estrogen conjugates excluding E2-17G caused by derivatisation procedure. Comparison with their absolute standards dissolving in neat organic solvent at the same concentration level $\left(1 \mu \mathrm{g} \mathrm{L}^{-1}\right)(n=3)$, the recoveries of E1-3S, E1-3G, E2-3S, E2-3G and E3-3S were 100.3\%, 97.2\%, $103.6 \%, 94.0 \%$ and $96.2 \%$, respectively (Fig. S4†). From this result, it was confirmed that conjugated estrogens without phenolic hydroxyl groups were inactive to DMIS and they were not affected on account of the mild reaction conditions.

\subsection{Chromatographic profiling of estrogens}

Given the basic characteristics of estrogen derivatives incurred by the structure of cationic moiety, positive polarity was preferable, as observed for the detection of dansyl estrogens. ${ }^{64}$ On the contrary, analysis of intact estrogen conjugates was more suitable to be undertaken under negative ESI due to their acidic nature. ${ }^{15}$

To achieve chromatographic separation and symmetric peak shapes for estrogens, organic solvents acetonitrile and methanol were compared. The same gradient program was utilised and sharper peak shape for analytes was found by using acetonitrile-water mobile phases than the methanol-water mobile phase. Additionally, estrogens especially their conjugated forms were found to yield higher intensity abundance when acetonitrile-water binary mobile phase was used, this was also observed by Sharanya Reddy et al. ${ }^{15}$ The disparity in response was possibly attributed to the lower viscosity owned by acetonitrile/water mixture than that of methanol-containing mixture, which may accelerate the formation of droplets with desirable size for subsequent evaporation of ions. ${ }^{68,69}$ 
Furthermore, the addition of $0.1 \%$ formic acid in mobile phase enhanced ESI response of derivatised estrogens than its absence, but intact estrogens were less affected. This may be due to the presence of acidic modifier facilitates ionisation of estrogen derivatives by helping protonation of analytes on their basic amine groups under positive polarity mode. ${ }^{31}$ However, in contrast to the use of formic acid, it has been stated that the involvement of alkaline buffer in mobile phase facilities the ionisation of acid compounds such as intact conjugated estrogens for greater resolution. ${ }^{63}$ As indicated in the literature, the mobile phase under basic condition often leads to premature failure of reverse-phase column arising from the increased solvability of silica with increasing $\mathrm{pH}^{70}$ Therefore, formic acid was more suitable to be added into mobile phase.

The analytes were injected on a capillary $\mathrm{C}_{18}$ column and LC-MS run was 30 min Fig. 3 shows LC chromatograms of targeted compounds spiked in post-extraction of $1000 \mathrm{~mL}$ surface water at concentration of $0.1 \mu \mathrm{g} \mathrm{L}^{-1}$. All targeted compounds were eluted between 8.99 and $14.83 \mathrm{~min}$. A chromatographic separation for the targeted compounds was challenging due to their rather similar chemical structures and physiochemical properties. It was consistent with the chromatographic profiles reported in previous methods for measurement of both free and conjugated estrogens, wherein up to 45 min gradient was used for each injection. ${ }^{28,63,65,71-73}$ Though the C18 column did not allow complete baseline separation of the chosen compounds, especially for E2-DMIS and EE2-DMIS as well as E1-3S and E2-3S, they could be differentiated by mass spectrometric signal by the different $\mathrm{m} / \mathrm{z}$ values of respective precursor ion and additionally by their specific fragmentation pathway upon $\mathrm{MS}^{2}$ fragmentation of their precursor ions.

If further optimisation of chromatographic separation is required, then it would be possible to test with stronger eluting mobile phases or/and even isocratic elution. Moreover, various flow rates of mobile phase and various LC columns could be further evaluated. It was reported that free estrogens derivatised with pyridine-3-sulfonyl chloride were better chromatographically separated using biphenyl stationary phase due to pi-pi and hydrophobic interactions, compared to $\mathrm{C}_{18}$ column on which only hydrophobic interaction and partitioning of molecules into the stationary phase normally occur. ${ }^{42}$ Moreover, LC run time for each injection could be further improved for increasing throughput via changing gradient program in future work.

\subsection{Optimisation of extraction method}

SPE is the most common technique used for a pre-concentration of endocrine disrupting chemicals from water or other more complex matrices. The factors possibly affecting recovery performance of extraction were evaluated for constructing a feasible method with satisfied accuracy and reproducibility.

3.4.1. Adsorbent for extraction. The selection SPE cartridges is the inception prior to proceeding with further optimisation. Adsorption capability mainly depended upon the materials of sorbents and properties of the interested chemicals. Free estrogens are weakly acidic and possess $\mathrm{p} K_{\mathrm{a}}$
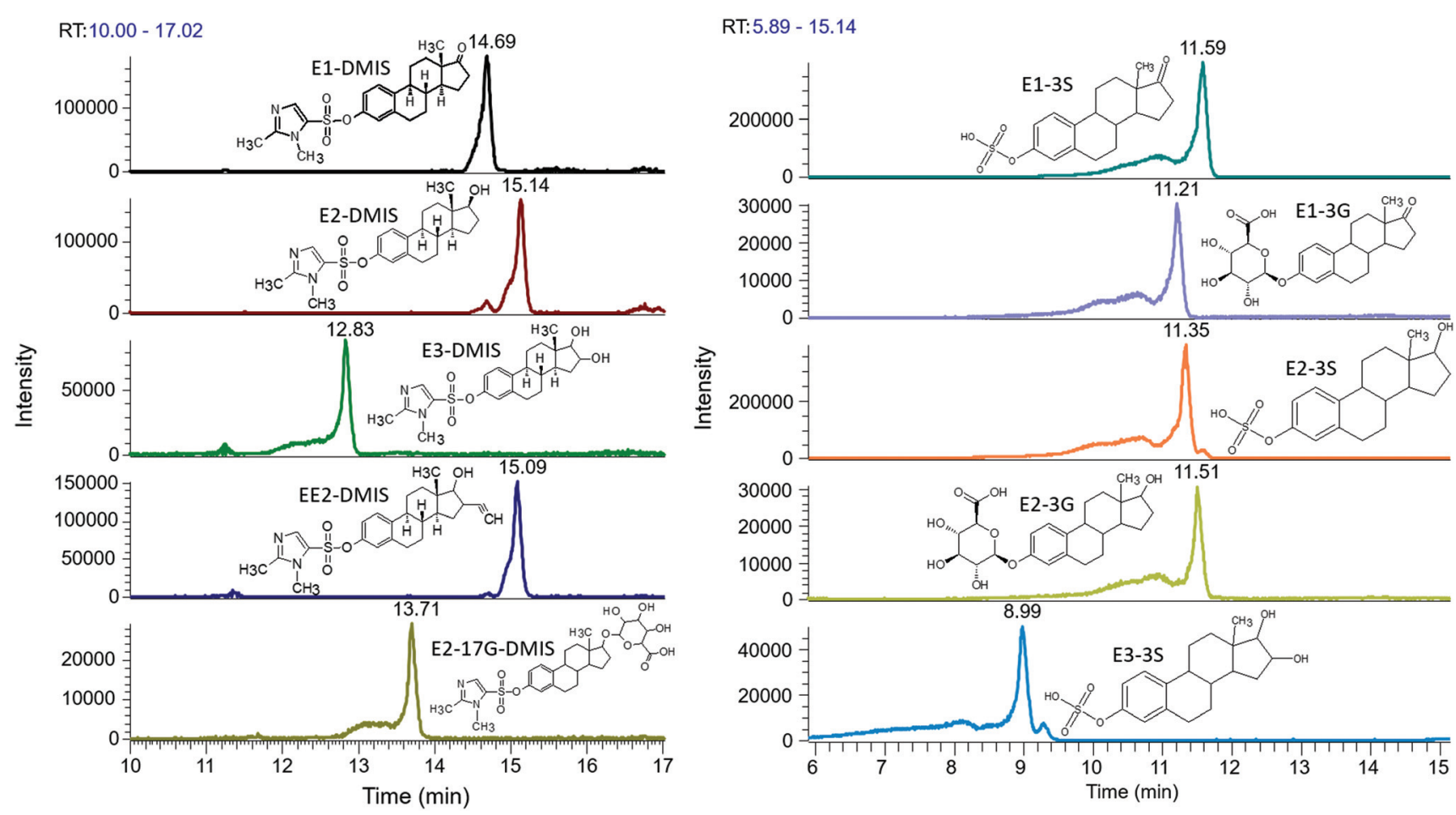

Fig. 3 Chromatograms of selective compounds spiked in extraction of surface water samples at concentration of $0.1 \mu g \mathrm{~L}^{-1}$. (i) Estrogen derivatives after reacting with DMIS under the positive ESI mode; (ii) intact conjugated estrogens under the negative ESI mode. 
around 10, which is much higher than that of sulphate and glucuronide estrogens. Referring to previous literature, the $\mathrm{p} K_{\mathrm{a}}$ of glucuronide conjugate was approximately 3 , and sulphate steroids are more acidic with $\mathrm{p} K_{\mathrm{a}}$ value $\sim-1.7 .{ }^{10,74}$ Due to the great difference between lipophilic free estrogens and hydrophilic estrogen conjugates, and highly broad range of polar and nonpolar property of them, reverse-phased absorbents were suitable for retaining targeted compounds due to the polymeric fillings. ${ }^{71}$ An Oasis HLB, Strata-X, Supel ${ }^{\mathrm{TM}}-$ Select HLB were selected and studied further by extracting $10 \mathrm{~mL}$ spiked Milli-Q water at concentration of $1 \mu \mathrm{g} \mathrm{L} \mathrm{L}^{-1}$. The result (Table 3) showed that Oasis HLB achieved satisfactory recoveries $(89.3 \%-102.8 \%)$ for chosen compounds reproducibility was with relative standard deviation (RSD) value less

Table 3 Recoveries rate ( \pm RSD \%) $(n=3)$ of MiliQ-water spiked $1 \mu \mathrm{L} \mathrm{L}^{-1}$ estrogen observed with three chosen different cartridges

\begin{tabular}{lccc}
\hline & $\begin{array}{l}\text { Oasis HLB } \\
\text { Recovery, \% } \\
\text { RSD, \%) }\end{array}$ & $\begin{array}{l}\text { Strata-X } \\
\text { Recovery, \% } \\
\text { RSD, \%) }\end{array}$ & $\begin{array}{l}\text { Supel } \\
\text { Recovery, \% } \\
\text { (RSD, \%) }\end{array}$ \\
\hline E1 & $96.4(2.3)$ & $93.8(7.74)$ & $89.4(6.06)$ \\
E2 & $98.9(2.01)$ & $75.2(5.61)$ & $74.5(4.11)$ \\
E3 & $92.3(1.88)$ & $68.3(1.79)$ & $87.6(1.93)$ \\
EE2 & $102.8(5.15)$ & $110.2(3.70)$ & $76.9(7.40)$ \\
E1-3S & $96.1(2.65)$ & $87.1(6.86)$ & $94.5(4.15)$ \\
E1-3G & $89.3(8.81)$ & $65.8(6.61)$ & $82.9(6.34)$ \\
E2-3S & $95.8(4.87)$ & $91.7(4.33)$ & $93.1(2.88)$ \\
E2-3G & $99.6(7.36)$ & $87.4(5.89)$ & $103.4(5.03)$ \\
E2-17G & $99.0(8.99)$ & $73.3(10.74)$ & $98.5(8.39)$ \\
E3-3S & $91.2(9.72)$ & $88.9(1.45)$ & $95.8(6.60)$
\end{tabular}

RSD: relative standard deviation (should be less than $20 \%$ ). ${ }^{75}$ than $9.72 \%$, having better performance than Stata-X (65.8\%-110.3\%) and Supel ${ }^{\mathrm{TM}}$-Select HLB cartridges $(74.5 \%-$ $103.3 \%)$. The versatility of Oasis HLB originates from its hydrophilic-lipophilic balance design in polymeric particles. Therefore, it is capable to affix moderately polar free estrogens having high $\log P$ value and much more hydrophilic estrogen conjugates which exhibit high polarity simultaneously. Considering the stability, the Oasis HLB cartridge was selected for this study.

3.4.2. $\mathrm{pH}$ and loading flow of water samples. The $\mathrm{pH}$ value of sample solution is known critical for enrichment of analytes as their acidic and alkaline properties. Ten $\mathrm{mL}$ Milli-Q water with spike of $1 \mu \mathrm{g} \mathrm{L} \mathrm{L}^{-1}$ estrogens had its $\mathrm{pH}$ adjusted to a range between 2.97 and 8.96 with $0.1 \mathrm{M} \mathrm{NaOH}$ or $\mathrm{H}_{2} \mathrm{SO}_{4}$ solution for evaluating the impacts of $\mathrm{pH}$ on recoveries of selected compounds. We observed that improved recoveries of free estrogens were achieved in samples with pH range of 2.97 to 7.01 , especially under the neutral condition. Further increase of alkalinity in solutions yielded poor recoveries for E1, E2 and EE2. The estrogen conjugates were given recoveries matched with requirement $80 \%-120 \%$ generally with the $\mathrm{pH}$ between 2.97 and 8.96 , but the losses of them were also noticed at $\mathrm{pH}$ 8.96 , in which the recovery of E3-3S had a remarkable reduction. It was possibly because that the alkaline solution induced the dissociation of acidic estrogens and hinder the binding to the SPE sorbents, leading to limited extraction efficiency. ${ }^{76}$ The recoveries of all estrogens at $\mathrm{pH} 7.01$ had a range of $81.3 \%-106.0 \%$ with acceptable coefficients of variation not over $10 \%$ excluding E3 at $13.0 \%$ and E3-3S at $11.2 \%$, showing the best performance among representative $\mathrm{pH}$ value chosen as demonstrated in Fig. 4.

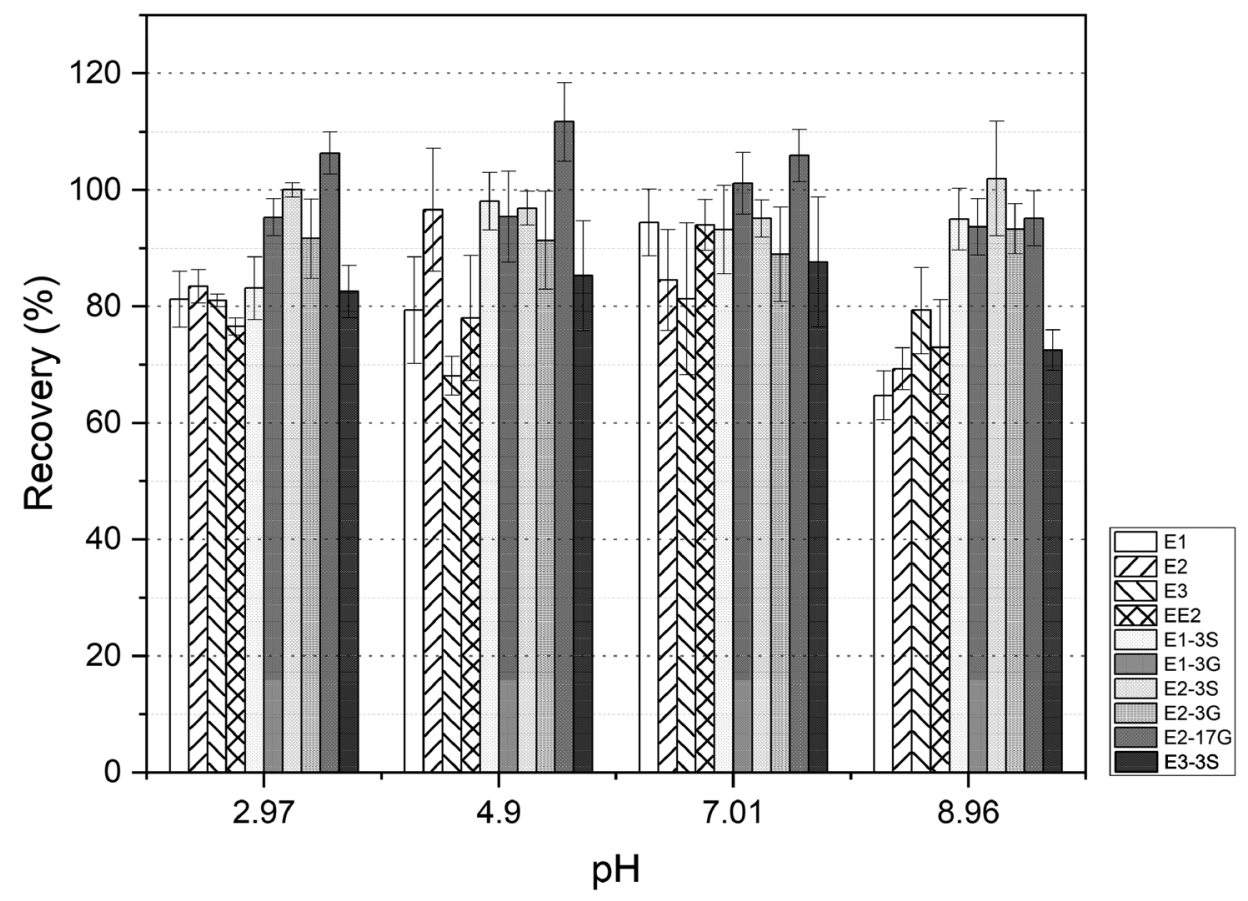

Fig. 4 Influence of $\mathrm{pH}$ of water samples on the recovery percentage of estrogens. 
Moreover, there is a study reporting that the co-extraction of humic acids in SPE sorbents is restricted substantially under neutral aqueous condition, then cleaner elutes with less interferences could be obtained. ${ }^{77}$

The influence of water samples through the SPE cartridges at load flow rate $\left(3 \mathrm{~mL} \mathrm{~min}^{-1}-5 \mathrm{~mL} \mathrm{~min}^{-1}\right)$ on the concentration efficiencies was studied. Theoretically, a slower flow rate allows adequate contact between the compounds and SPE sorbents, so a higher recovery percentage could be obtained. However, the test results demonstrated that negligible improvement was discovered on the recovery of targeted compounds over the chosen flow rate $3 \mathrm{~mL} \min ^{1}-5 \mathrm{~mL} \mathrm{~min}^{-1}$. Consequently, the adoption of $5 \mathrm{~mL} \mathrm{~min}^{-1}$ was finally decided in order to increase throughput.

3.4.3. Optimisation of the elution solvents. As another crucial factor that decides the optimisation of SPE procedure, the evaluation of eluent solvents was carried out with $10 \mathrm{~mL}$ Milli-Q water with a spike of $1 \mu \mathrm{g} \mathrm{L^{-1 }}$ estrogens. Eight $\mathrm{mL}$ organic solvents [methanol, acetone, ethyl acetate, $5: 1(\mathrm{v}: \mathrm{v})$ ethyl acetate/methanol mixture, $3: 2: 5$ ( $\mathrm{v}: \mathrm{v}: \mathrm{v})$ dichloromethane (DCM)/acetone/methanol ternary mixture and methanol containing $5 \%$ acetonitrile] were assayed, the results revealed that conjugated estrogens were poorly recovered using either ethyl acetate or 5:1 ethyl acetate/methanol binary solvent, especially E3-3S, which exhibited low recovery percentage from below $10 \%$ to $65.4 \%$. This was mainly attributed to the high polarity of conjugated estrogens, the elution strength of elutes which were dominanted by the ethyl acetate component may not be adequate to release them from the HLB sor- bents. Significant improved recoveries with a span of from $83.2 \%$ to $118.9 \%$ were obtained when eluted by methanol, mixed solution of $3: 2: 5 \mathrm{DCM} /$ acetone/methanol and methanol including $5 \%$ acetonitrile for both free and conjugated estrogens. Therefore, the final decision of superior elute was made by further testing the recovery of targeted chemicals in water samples with increasing volume to $500 \mathrm{~mL}$ and $1000 \mathrm{~mL}$.

The extraction efficiencies of estrogens in aqueous solution with various loading volumes using these three elution solvents are summarised in Fig. 5. The results revealed that using $100 \%$ methanol achieved the optimum pre-concentration performance in all three volume levels of water samples with the

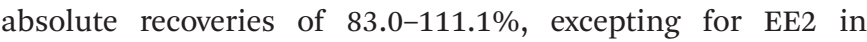
$1000 \mathrm{~mL}$ sample, which yielded a relatively poor extraction efficiency of $75.9 \%$ with the RSDs less than $15 \%$ overall. Therefore, methanol was employed to elute estrogens off the cartridges in this study.

3.4.4. Washing solution. It is known that interferences originated from natural samples retain in SPE sorbents and are potentially co-eluted with targeted compounds together, leading to ion suppression to some extent. Therefore, the washing step following loading samples closely was tested using spiked natural lake water at the concentration level of $1 \mu \mathrm{g} \mathrm{L} \mathrm{L}^{-1}$ in triplicate $(n=3)$. The composition of methanol in Milli-Q water from $0 \%$ to $10 \%$ with an increment of $5 \%$ was studied for eliminating matrix but not compromising extraction performance. The results show that there was a reduction of recoveries of estrogens when there was increasing percentage of methanol in washing solution.

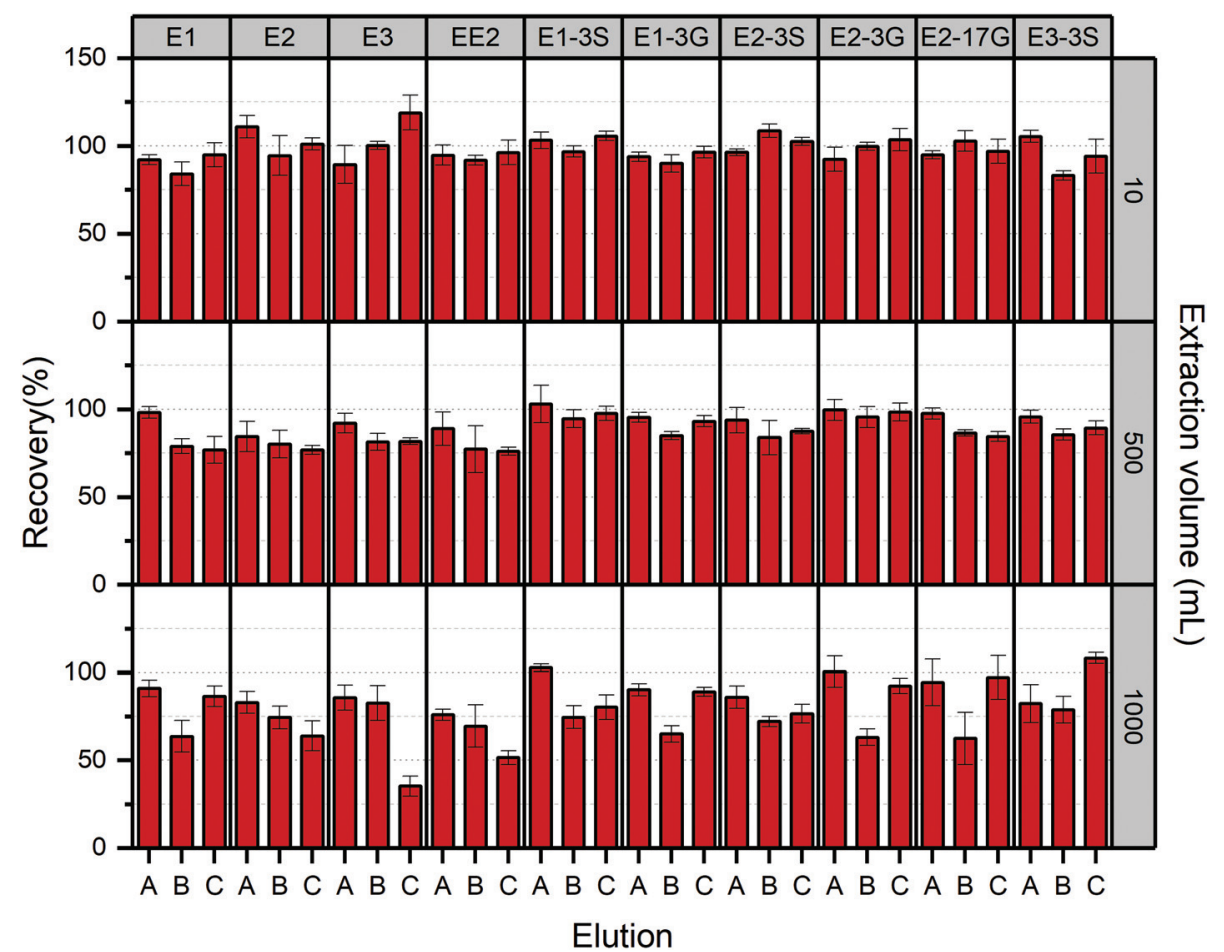

Fig. 5 Recovery efficiencies (\%) and RSD (\%) of estrogens in aqueous solution with various loading volume (10, 500 and $1000 \mathrm{~mL})$ using these three elution solvents (A) methanol; (B) DCM : acetone : methanol, $3: 2: 5$ ( $: v: v)$; (C) methanol with $5 \%$ acetonitrile. 
Thus, the $8 \mathrm{~mL}$ of Milii-Q water free of methanol was suitable for washing away interferences without a loss of targeted compounds. The common application of pure water to rinse interfering substances away from cartridges was also reported in previous studies. ${ }^{36-39}$ According to the literature, the composition of washing solution matters more to the efficacy to remove impurities adsorbed by cartridges than the volume factor.

The further optimisation of the volume and composition of washing solution for cleaning up enriched cartridges is required if larger sample volume or complicated matrix is studied.

\subsection{Method validation}

The validation of the established method for determining both free and conjugated estrogens using natural surface water is summarised in Table 4 .

To investigate the linearity of the established method, calibration curves for all estrogens were constructed by using matrix matched solutions over a wide concentration to $1000 \mu \mathrm{g}$ $\mathrm{L}^{-1}$, covering their instrumental quantification limit (IQL). The correlation coefficient $R^{2}$ for each analyte greater than 0.99 was obtained in both standard diluents and surface water.

The method intra-day and inter-day repeatability was evaluated by the relative standard deviations (RSDs) of intra-day and inter-day which were between $2.84 \%$ and $11.73 \%$, the good repeatability and stability of the established method were accounted for, meeting the requirements for the following analysis.

The selectivity of the modified methodology was estimated by analysing non-dosed matrix and surface water sample dosed at IQL values which are summarised in Table S2. $\dagger$ Using E3-DMIS as a surrogate, typical reconstructed ion chromatograms for $m / z 447.3$ of non-spiked and spiked matrix are presented in Fig. S5(A and B), $\dagger$ which shows no significant interferences occurred at the retention time for targeted estrogens in chromatogram window of non-dosed sample using the $\mathrm{m} / \mathrm{z}$ value of precursors. Moreover, the characteristic fragment ions in $\mathrm{MS}^{2}$ spectrum were also capable of identifying analytes of interest from other co-eluted matrix components. Thus, it was concluded that the established LC-MS methodology exhibited satisfactory selectivity for the determination of estrogens in natural water.

As previously reported, the components in natural matrix are prone to result in either ion suppression or enhancement of response for analytes under electrospray conditions, which potentially could affect an accuracy and reproducibility of results. ${ }^{53}$ Consequently, it is pivotal to take matrix effects into account, especially when quantification is undertaken using an external calibration curves. Ciofi, Fibbi $^{78}$ evaluated the matrix effect in river and lake waters by the comparison of peak areas for selective compounds in spiked water samples with those acquired from spiked Milli-Q water. In our study, matrix effects were estimated based on the post-addition method, ${ }^{79}$ using the ratio of the calibration curve slopes for estrogen standard solutions spiked into extraction of surface water matrix and neat dilution solvent. The occurrence of ion suppression was evidenced as the matrix effect was larger than zero. However, it was also found that the matrix effect was at a low level between $8.32 \%$ and $22.69 \%$. According to the results obtained in other studies, the matrix effect was noticed to be related to the values of dissolved organic carbon (DOC) and chemical oxygen demand (COD) for samples; and the higher matrix effect was attained within samples owning higher DOC or COD. ${ }^{78}$ In addition to the aqueous chemical condition of samples, the types of ionisation source used also contributes to the discrepancy of signals caused by matrix. ${ }^{64}$ Through the comparison between analytes, we found that the glucuronide form of estrogens suffered the most severe matrix effects than other analytes, which is possibly explained by their high hydrophilicity, resulting in restricted separation from the co-eluting matrix along with the LC column. The similar observation was also achieved for investigating the impact of waste water matrix on glucuronide estrogens, while the higher loss of signal was derived due to the presence of more complicated interferences in waste water usually. ${ }^{71}$

The low (50 ng L $\left.{ }^{-1}\right)$, middle (100 ng L $\left.{ }^{-1}\right)$ and high (1000 ng $\mathrm{L}^{-1}$ ) three concentration levels were spiked into natural surface

Table 4 Linear range and validation results of estrogens in surface water using LC-MS

Surface water

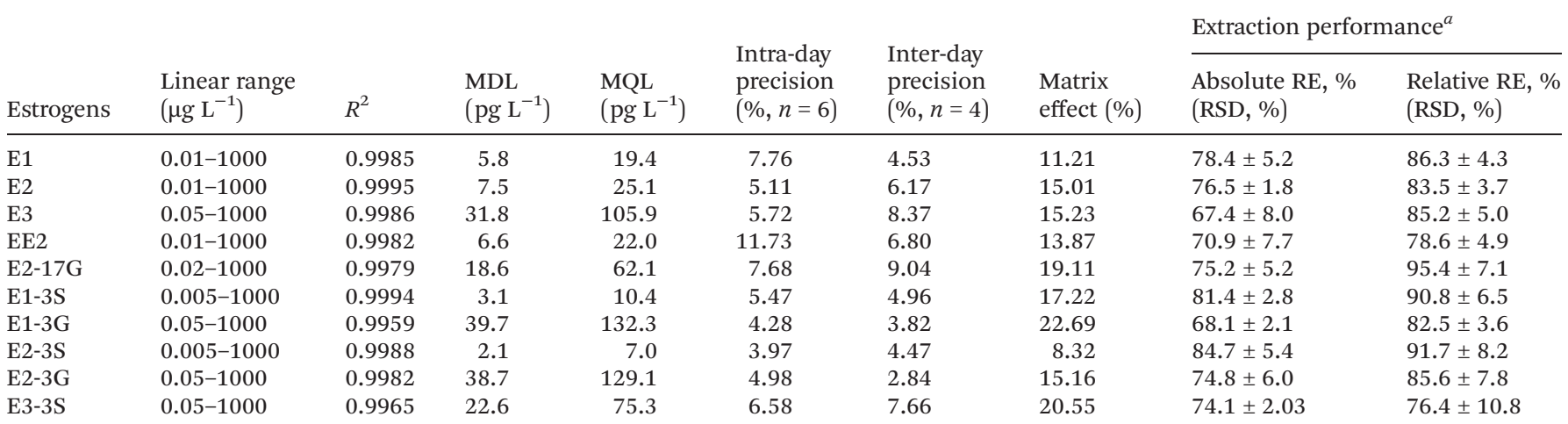

${ }^{a}$ Average recovery of estrogens in $1000 \mathrm{~mL}$ natural surface water (spiked at three levels concentration: 50,100 and $1000 \mathrm{ng} \mathrm{\textrm {L } ^ { - 1 }}$ ). 
water for checking the recovery performance of selected estrogens. In Table 4 are presented only the average recovery of estrogens in surface water, satisfactory relative recoveries were still obtained for the majority of selected compounds, which met the requirement from $80 \%$ to $120 \%$ with $\mathrm{RSD}<10.8 \%$, except for EE2 and E1-3G, having extraction percentage of $78.6 \%$ and $76.4 \%$, respectively.

The IDLs, IQLs, MDLs and MQLs for all chosen estrogens are presented in Table $\mathrm{S} 2 \dagger$ and Table 4 . The highest response was observed for sulphate estrogens, especially for E1-3S and E2-3S with low IDLs of $2.5 \mathrm{ng} \mathrm{\textrm {L } ^ { - 1 }}$ and $1.8 \mathrm{ng} \mathrm{\textrm {L } ^ { - 1 }}$, respectively. However, another sulphate compound E3-3S yielded a poor signal, obtaining an IDL at $16.8 \mathrm{ng} \mathrm{\textrm {L } ^ { - 1 }}$. Derivatives of free estrogens also produced high signal, whose IDLs ranged from

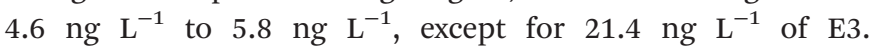
Glucuronide-containing estrogens had the poorest sensitivity

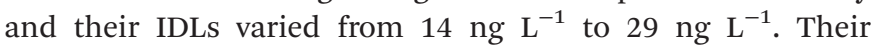
corresponding IQLs were calculated and the lowest concentration point of the calibration curve was settled accordingly. MQLs found based on an enrichment factor of 1000 were at low picogram to nanogram per litre levels and in a range of 7-132.3 $\mathrm{pg} \mathrm{L}^{-1}$ for analytes.

\subsection{Application to environmental samples}

An optimised/validated method was employed to determine the content of free and conjugated estrogens in natural water collected from the Regent's Park lake in London, UK. Using the standard addition method, known amounts of estrogens standards were added into $1000 \mathrm{~mL}$ water samples for calculating the original concentrations of analytes by calculating an $\mathrm{X}$-intercept value of corresponding calibration curve.

Table 5 summarises the maximum and average concentrations of free and conjugated estrogens measured in 7 water samples. Notably, the contents of free estrogens were significantly higher than their conjugated compounds, in which E1 was found the most abundant compared with E2 and E3, having a maximum concentration of $0.86 \mathrm{ng} \mathrm{L}^{-1}$ and average concentration of $0.21 \mathrm{ng} \mathrm{L^{-1 }}$ in 7 samples. These concentrations are consistent with those reported in previous studies

Table 5 Concentration of free and conjugated estrogens in natural water collected from Regent's Park lake

\begin{tabular}{lll}
\hline & \multicolumn{2}{l}{ Surface water $(n=7)$} \\
\cline { 2 - 3 } & $\begin{array}{l}\text { Concentration max } \\
\left(\mathrm{ng} \mathrm{L}^{-1}\right)\end{array}$ & $\begin{array}{l}\text { Concentration mean } \\
\left(\mathrm{ng} \mathrm{L}^{-1}\right)\end{array}$ \\
\hline E1 & 0.86 & 0.32 \\
E2 & 0.55 & 0.21 \\
E3 & 0.48 & 0.19 \\
EE2 & $<\mathrm{MQL}$ & $<\mathrm{MQL}$ \\
E2-17G & $<\mathrm{MQL}$ & $<\mathrm{MQL}$ \\
E1-3S & 0.17 & 0.11 \\
E1-3G & $<\mathrm{MQL}$ & $<\mathrm{MQL}$ \\
E2-3S & 0.09 & 0.05 \\
E2-3G & $<\mathrm{MQL}$ & $<\mathrm{MQL}$ \\
E3-3S & $<\mathrm{MQL}$ & $<\mathrm{MQL}$
\end{tabular}

for surface water. Williams, Johnson ${ }^{80}$ detected E1 at concentration of $<0.4-2.5 \mathrm{ng} \mathrm{L}{ }^{-1}$ and $\mathrm{E} 2$ of $<0.4-0.8 \mathrm{ng} \mathrm{\textrm {L } ^ { - 1 }}$ in River Nene and River Lea. Owing to a great population of birds dwelling in the Regent's Park, the occurrence of estrogens was expected in the lake water because of the possible contamination by excretion from birds. Moreover, it was hypothesised that the residue of estrogens could reach to collection sites via aquifers connecting to the run-off since the previous reported presence of E1, E2 and E1-3S profiled at concentrations (0.4-120, 0.2-45 and 1-4 $\mathrm{ng} \mathrm{L}^{-1}$, respectively) in ground water. ${ }^{81}$ As the precursors of free estrogens, conjugated estrogens are hydrolysed gradually with the presence of microorganisms. ${ }^{82}$ Furthermore, it has been evidenced before that a majority free estrogens are biodegraded via E1. ${ }^{83}$ The amount of EE2 in sample was found less than its quantification limit. That is because, as a synthetic constituent mainly applied for birth control tablets, EE2 is hardly found in litter of birds, consequently it was not detected in the near-by sampling sites. Glucuronides were not detectable in natural water. In contrast, the prevalence of sulphates was observed, which was attributed to lower MDLs the sulphates possessed; additionally, the transformation of glucuronides to free estrogens was inferred in natural water while the sulphates were more recalcitrant to biodegradation induced by microorganisms, as reported prior to this study. ${ }^{84}$

\section{Conclusion}

In this study, we modified a sensitive LC-MS method based on derivatisation with DMIS which was previously used to discriminate E2, testosterone and dihydrotestosterone in serum, ${ }^{35}$ to detect, for the first time, free and conjugated estrogens at trace level in natural water. The occurrence of derivatisation reaction was significantly favourable for enhancing mass spectrometric signal under ESI conditions of free estrogens. Derivatives affixing DMIS label were capable of providing specific structural characterisation for the confirmation of chosen compounds using MS/MS, from which structurally isomers E2-17G and E2-3G were identified due to the selectivity of derivatisation reaction, alleviating the susceptibility of determination in the presence of interferences. Following a feasible SPE procedure, the enrichment of analytes from samples was achieved using Oasis HLB sorbent, and impurities were washed away with Milli-Q water followed by elution using $8 \mathrm{~mL}$ methanol. Method quantification limits for all estrogens ranging from 7.0 to $132.3 \mathrm{pg} \mathrm{L}^{-1}$ in surface water were acquired by validation of the established methodology.

The practicability of this method was demonstrated by profiling estrogens in surface water collected from a lake of the Regent's Park. Only EE2 and E3-3S were not found in samples, and other compounds were detected. However, their concentrations levels were differential, for example sulphates were significantly prevalent than glucuronide estrogens. Our results confirmed the capability of monitoring estrogens in surface water using this modified methodology. 


\section{Author contributions}

Fan Huang-Conceptualization, methodology, writing - original draft. Kersti Karu-Methodology, writing - review \& editing. Luiza C. Campos-Supervision, administration of project, writing - review \& editing.

\section{Conflicts of interest}

There are no known conflicts to declare.

\section{Acknowledgements}

The authors would like to thank Mr Emmanuel Samuel for his help on troubleshooting for LC-MS system at the beginning of this project. Specially, authors are grateful for the water collection permission from the Royal Parks. This research did not receive any specific grant from funding agencies in the public, commercial, or not-for-profit sectors.

\section{References}

1 S. X. Goh, et al., Online solid phase extraction with liquid chromatography-tandem mass spectrometry for determination of estrogens and glucocorticoids in water, J. Chromatogr. A, 2016, 1465, 9-19.

2 Q. Chen, et al., A new pretreatment and improved method for determination of selected estrogens in high matrix solid sewage samples by liquid chromatography mass spectrometry, Microchem. J., 2012, 104, 49-55.

3 L. Wang, et al., Assessing estrogenic activity in surface water and sediment of the Liao River system in northeast China using combined chemical and biological tools, Environ. Pollut., 2011, 159(1), 148-156.

4 B. Banihashemi and R. L. Droste, Sorption-desorption and biosorption of bisphenol A, triclosan, and 17alpha-ethinylestradiol to sewage sludge, Sci. Total Environ., 2014, 487, 813-821.

5 D. L. Cheng, et al., Bioprocessing for elimination antibiotics and hormones from swine wastewater, Sci. Total Environ., 2018, 621, 1664-1682.

6 B. Xu, et al., UV photoconversion of environmental oestrogen diethylstilbestrol and its persistence in surface water under sunlight, Water Res., 2017, 127, 77-85.

7 M. Clara, et al., Adsorption of bisphenol-A, 17 beta-estradiole and 17 alpha-ethinylestradiole to sewage sludge, Chemosphere, 2004, 56(9), 843-851.

8 L. Ma and S. R. Yates, Degradation and metabolite formation of 17 ss-estradiol-3-glucuronide and 17ss-estradiol3-sulphate in river water and sediment, Water Res., 2018, 139, 1-9.

9 T. Fukuhara, et al., Adsorbability of estrone and 17betaestradiol in water onto activated carbon, Water Res., 2006, 40(2), 241-248.
10 F. Qin, et al., Hydrophilic interaction liquid chromatography- tandem mass spectrometry determination of estrogen conjugates in human urine, Anal. Chem., 2008, 80(9), 3404-3411.

$11 \mathrm{X} . \mathrm{Xu}$, et al., Measuring seven endogenous ketolic estrogens simultaneously in human urine by high-performance liquid chromatography- mass spectrometry, Anal. Chem., 2004, 76(19), 5829-5836.

12 S. Poschner, et al., Simultaneous quantification of estrogens, their precursors and conjugated metabolites in human breast cancer cells by LC-HRMS without derivatization, J. Pharm. Biomed. Anal., 2017, 138, 344-350.

$13 \mathrm{X} . \mathrm{Xu}$, et al., Measuring fifteen endogenous estrogens simultaneously in human urine by high-performance liquid chromatography-mass spectrometry, Anal. Chem., 2005, 77(20), 6646-6654.

$14 \mathrm{X} . \mathrm{Xu}$, et al., Quantitative measurement of endogenous estrogens and estrogen metabolites in human serum by liquid chromatography-tandem mass spectrometry, Anal. Chem., 2007, 79(20), 7813-7821.

15 S. Reddy, C. R. Iden and B. J. Brownawell, Analysis of steroid conjugates in sewage influent and effluent by liquid chromatography-tandem mass spectrometry, Anal. Chem., 2005, 77(21), 7032-7038.

16 A. K. Singh, et al., Quantitative analysis of conjugated and free estrogens in swine manure: solutions to overcome analytical problems due to matrix effects, J. Chromatogr. A, 2013, 1305, 203-212.

17 H. Fang, et al., Structure- activity relationships for a large diverse set of natural, synthetic, and environmental estrogens, Chem. Res. Toxicol., 2001, 14(3), 280-294.

18 D. R. Griffith, et al., Measuring Free, Conjugated, and Halogenated Estrogens in Secondary Treated Wastewater Effluent, Environ. Sci. Technol., 2014, 48(5), 2569-2578.

$19 \mathrm{~V}$. Kumar, et al., The arrival and discharge of conjugated estrogens from a range of different sewage treatment plants in the UK, Chemosphere, 2011, 82(8), 1124-1128.

20 R. P. Suri, T. S. Singh and R. F. Chimchirian, Effect of process conditions on the analysis of free and conjugated estrogen hormones by solid-phase extraction-gas chromatography/mass spectrometry (SPE-GC/MS), Environ. Monit. Assess., 2012, 184(3), 1657-1669.

21 G. D'ascenzo, et al., Fate of natural estrogen conjugates in municipal sewage transport and treatment facilities, Sci. Total Environ., 2003, 302(1-3), 199-209.

22 A. Gonzalez, J. Avivar and V. Cerda, Estrogens determination in wastewater samples by automatic in-syringe dispersive liquid-liquid microextraction prior silylation and gas chromatography, J. Chromatogr. A, 2015, 1413, 1-8.

23 T. Y. Fang, et al., Analytical techniques for steroid estrogens in water samples - A review, Chemosphere, 2016, 165, 358368.

24 W. Dai, et al., Comprehensive and highly sensitive urinary steroid hormone profiling method based on stable isotopelabeling liquid chromatography-mass spectrometry, Anal. Chem., 2012, 84(23), 10245-10251. 
25 R. Su, et al., Application of multiwall carbon nanotubesbased matrix solid phase dispersion extraction for determination of hormones in butter by gas chromatography mass spectrometry, J. Chromatogr. A, 2011, 1218(31), 50475054.

$26 \mathrm{X}$. Xu, et al., Stable isotope dilution high-performance liquid chromatography-electrospray ionization mass spectrometry method for endogenous 2-and 4-hydroxyestrones in human urine, J. Chromatogr. B: Anal. Technol. Biomed. Life Sci., 2002, 780(2), 315-330.

$27 \mathrm{X}$. Li and A. A. Franke, Improved profiling of estrogen metabolites by orbitrap LC/MS, Steroids, 2015, 99(Pt A), 8490.

28 J. Tso and D. S. Aga, A systematic investigation to optimize simultaneous extraction and liquid chromatography tandem mass spectrometry analysis of estrogens and their conjugated metabolites in milk, J. Chromatogr. A, 2010, 1217(29), 4784-4795.

29 Y. Nie, et al., Determination of endocrine-disrupting chemicals in the liquid and solid phases of activated sludge by solid phase extraction and gas chromatographymass spectrometry, J. Chromatogr. A, 2009, 1216(42), 70717080 .

30 Z. Fan, et al., Detection and occurrence of chlorinated byproducts of bisphenol a, nonylphenol, and estrogens in drinking water of china: comparison to the parent compounds, Environ. Sci. Technol., 2013, 47(19), 10841-10850.

31 M. R. Anari, et al., Derivatization of ethinylestradiol with dansyl chloride to enhance electrospray ionization: application in trace analysis of ethinylestradiol in rhesus monkey plasma, Anal. Chem., 2002, 74(16), 4136-4144.

32 R. E. Nelson, et al., Liquid chromatography-tandem mass spectrometry assay for simultaneous measurement of estradiol and estrone in human plasma, Clin. Chem., 2004, 50(2), 373-384.

33 Y. H. Lin, C. Y. Chen and G. S. Wang, Analysis of steroid estrogens in water using liquid chromatography/tandem mass spectrometry with chemical derivatizations, Rapid Commun. Mass Spectrom., 2007, 21(13), 1973-1983.

34 A. M. Faqehi, et al., Derivatization of estrogens enhances specificity and sensitivity of analysis of human plasma and serum by liquid chromatography tandem mass spectrometry, Talanta, 2016, 151, 148-156.

35 P. Keski-Rahkonen, et al., Measurement of Estradiol in Human Serum by LC-MS/MS Using a Novel EstrogenSpecific Derivatization Reagent, Anal. Chem., 2015, 87(14), 7180-7186.

36 M. J. López de Alda and D. Barceló, Use of solid-phase extraction in various of its modalities for sample preparation in the determination of estrogens and progestogens in sediment and water, J. Chromatogr., A, 2001, 938(1), 145153.

37 S. Rodriguez-Mozaz, M. J. Lopez de Alda and D. Barceló, Picogram per liter level determination of estrogens in natural waters and waterworks by a fully automated on-line solid-phase extraction-liquid chromatography-electrospray tandem mass spectrometry method, Anal. Chem., 2004, 76(23), 6998-7006.

$38 \mathrm{~J} . \mathrm{Hu}, \mathrm{H}$. Zhang and $\mathrm{H}$. Chang, Improved method for analyzing estrogens in water by liquid chromatography-electrospray mass spectrometry, J. Chromatogr., A, 2005, 1070(1-2), 221-224.

39 T. Trinh, et al., Simultaneous determination of estrogenic and androgenic hormones in water by isotope dilution gas chromatography-tandem mass spectrometry, J. Chromatogr. A, 2011, 1218(12), 1668-1676.

40 Y. Filali-Meknassi, et al., Quantification of steroid sex hormones using solid-phase extraction followed by liquid chromatography-mass spectrometry, Water Environ. Res., 2007, 79(6), 687-696.

41 A. Laganà, et al., Analytical methodologies for determining the occurrence of endocrine disrupting chemicals in sewage treatment plants and natural waters, Anal. Chim. Acta, 2004, 501(1), 79-88.

42 A. Glineur, et al., Trace analysis of estrogenic compounds in surface and groundwater by ultra high performance liquid chromatography-tandem mass spectrometry as pyridine-3-sulfonyl derivatives, J. Chromatogr. A, 2018, 1534, 43-54.

43 Food and D. Administration, Guidance for industry: bioanalytical method validation. 2001, Rockville, MD, USA, 2016.

44 D. C. Harris, Quantitative chemical analysis, Macmillan, 2010.

45 Y. Nie, et al., Determination of endocrine-disrupting chemicals in the liquid and solid phases of activated sludge by solid phase extraction and gas chromatographymass spectrometry, J. Chromatogr. A, 2009, 1216(42), 70717080.

46 T. A. Ternes, et al., Determination of estrogens in sludge and sediments by liquid extraction and GC/MS/MS, Anal. Chem., 2002, 74(14), 3498-3504.

47 D. R. Baker and B. Kasprzyk-Hordern, Multi-residue analysis of drugs of abuse in wastewater and surface water by solid-phase extraction and liquid chromatography-positive electrospray ionisation tandem mass spectrometry, J. Chromatogr. A, 2011, 1218(12), 1620-1631.

48 O. Gonzalez, et al., Bioanalytical chromatographic method validation according to current regulations, with a special focus on the non-well defined parameters limit of quantification, robustness and matrix effect, J. Chromatogr. A, 2014, 1353, 10-27.

49 N. Singh, et al., Development and validation of novel LCMS/MS method for determination of Lusutrombopag in rat plasma and its application to pharmacokinetic studies, Arabian J. Chem., 2020, 13(2), 4162-4169.

50 G. J. Van Berkel, S. A. McLuckey and G. L. Glish, Preforming ions in solution via charge-transfer complexation for analysis by electrospray ionization mass spectrometry, Anal. Chem., 1991, 63(18), 2064-2068.

51 H. Tomšíková, et al., High-sensitivity analysis of femalesteroid hormones in environmental samples, TrAC, Trends Anal. Chem., 2012, 34, 35-58. 
52 D. W. Newton and R. B. Kluza, pKa values of medicinal compounds in pharmacy practice, Drug Intell. Clin. Pharm., 1978, 12(9), 546-554.

53 A. Salvador, et al., On-line solid-phase extraction with onsupport derivatization for high-sensitivity liquid chromatography tandem mass spectrometry of estrogens in influent/ effluent of wastewater treatment plants, J. Chromatogr. A, 2007, 1145(1-2), 102-109.

54 D. B. Robb, T. R. Covey and A. P. Bruins, Atmospheric pressure photoionization: an ionization method for liquid chromatography- mass spectrometry, Anal. Chem., 2000, 72(15), 3653-3659.

55 S.-I. Nakao, et al., Chemistry of Amine-Based CO 2 Capture, in Advanced $\mathrm{CO}_{2}$ Capture Technologies, 2019, Springer, pp. $3-22$.

56 L. Xu and D. C. Spink, 1, 2-Dimethylimidazole-4-sulfonyl chloride, a novel derivatization reagent for the analysis of phenolic compounds by liquid chromatography electrospray tandem mass spectrometry: Application to 1-hydroxypyrene in human urine, J. Chromatogr. B: Anal. Technol. Biomed. Life Sci., 2007, 855(2), 159-165.

57 M. Lampinen-Salomonsson, et al., Differentiation of estriol glucuronide isomers by chemical derivatization and electrospray tandem mass spectrometry, Rapid Commun. Mass Spectrom., 2006, 20(9), 1429-1440.

58 M. L. Salomonsson, U. Bondesson and M. Hedeland, Structural evaluation of the glucuronides of morphine and formoterol using chemical derivatization with 1,2-dimethylimidazole-4-sulfonyl chloride and liquid chromatography/ ion trap mass spectrometry, Rapid Commun. Mass Spectrom., 2008, 22(17), 2685-2697.

59 L. Xu and D. C. Spink, Analysis of steroidal estrogens as pyridine-3-sulfonyl derivatives by liquid chromatography electrospray tandem mass spectrometry, Anal. Biochem., 2008, 375(1), 105-114.

60 M. Lampinen Salomonsson, U. Bondesson and M. Hedeland, In vitro formation of phase I and II metabolites of propranolol and determination of their structures using chemical derivatization and liquid chromatographytandem mass spectrometry, J. Mass Spectrom., 2009, 44(5), 742-754.

61 C. Djerassi, et al., Mass Spectrometry in Structural and Stereochemical Problems. XIV. 1 Steroids with One or Two Aromatic Rings 2, J. Am. Chem. Soc., 1962, 84(23), 45444552.

62 S. Reddy, C. R. Iden and B. J. Brownawell, Analysis of steroid conjugates in sewage influent and effluent by liquid chromatography-tandem mass spectrometry, Anal. Chem., 2005, 77(21), 7032.

63 N. L. Kuehnbaum and P. Britz-McKibbin, Comprehensive profiling of free and conjugated estrogens by capillary electrophoresis-time of flight/mass spectrometry, Anal. Chem., 2011, 83(21), 8063-8068.

64 G. W. Lien, C. Y. Chen and G. S. Wang, Comparison of electrospray ionization, atmospheric pressure chemical ionization and atmospheric pressure photoionization for deter- mining estrogenic chemicals in water by liquid chromatography tandem mass spectrometry with chemical derivatizations, J. Chromatogr. A, 2009, 1216(6), 956-966.

65 A. Nieto, et al., Determination of natural and synthetic estrogens and their conjugates in sewage sludge by pressurized liquid extraction and liquid chromatography-tandem mass spectrometry, J. Chromatogr. A, 2008, 1213(2), 224230.

66 A. C. Naldi, et al., Analysis of steroid hormones and their conjugated forms in water and urine by on-line solid-phase extraction coupled to liquid chromatography tandem mass spectrometry, Chem. Cent. J., 2016, 10, 30.

67 A. Gentili, et al., Analysis of free estrogens and their conjugates in sewage and river waters by solid-phase extraction then liquid chromatography-electrospray-tandem mass spectrometry, Chromatographia, 2002, 56(1-2), 25.

68 T. Benijts, et al., Analysis of estrogenic contaminants in river water using liquid chromatography coupled to ion trap based mass spectrometry, Rapid Commun. Mass Spectrom., 2002, 16(14), 1358-1364.

69 J. Yao, et al., The Effect of Oil Viscosity on Droplet Generation Rate and Droplet Size in a T-Junction Microfluidic Droplet Generator, Micromachines, 2019, 10(12), 808.

70 J. Atwood, G. Schmidt and W. Slavin, Improvements in liquid chromatography column life and method flexibility by saturating the mobile phase with silica, J. Chromatogr., A, 1979, 171, 109-115.

71 D. R. Griffith, et al., Measuring free, conjugated, and halogenated estrogens in secondary treated wastewater effluent, Environ. Sci. Technol., 2014, 48(5), 2569-2578.

72 C. van der Berg, et al., Development and validation of LC-ESI-MS/MS methods for quantification of 27 free and conjugated estrogen-related metabolites, Anal. Biochem., 2020, 590, 113531.

73 M. Kuster, et al., Analysis of phytoestrogens, progestogens and estrogens in environmental waters from Rio de Janeiro (Brazil), Environ. Int., 2009, 35(7), 997-1003.

74 P. He and D. S. Aga, Comparison of GC-MS/MS and LC-MS/ MS for the analysis of hormones and pesticides in surface waters: advantages and pitfalls, Anal. Methods, 2019, 11(11), 1436-1448.

75 W. Ben, et al., Simultaneous determination of sulfonamides, tetracyclines and tiamulin in swine wastewater by solid-phase extraction and liquid chromatography-mass spectrometry, J. Chromatogr. A, 2008, 1202(2), 173-180.

76 R. Liu, J. L. Zhou and A. Wilding, Simultaneous determination of endocrine disrupting phenolic compounds and steroids in water by solid-phase extraction-gas chromatography-mass spectrometry, J. Chromatogr. A, 2004, 1022(1-2), 179-189.

77 M.-C. Hennion, Solid-phase extraction: method development, sorbents, and coupling with liquid chromatography, J. Chromatogr., A, 1999, 856(1-2), 3-54.

78 L. Ciofi, et al., Fully-automated on-line solid phase extraction coupled to high-performance liquid chromatography- 
tandem mass spectrometric analysis at sub-ng/L levels of selected estrogens in surface water and wastewater, J. Chromatogr. A, 2013, 1283, 53-61.

79 J. Kang, L. A. Hick and W. E. Price, Using calibration approaches to compensate for remaining matrix effects in quantitative liquid chromatography/electrospray ionization multistage mass spectrometric analysis of phytoestrogens in aqueous environmental samples, Rapid Commun. Mass Spectrom., 2007, 21(24), 4065-4072.

80 R. J. Williams, et al., Steroid estrogens profiles along river stretches arising from sewage treatment works discharges, Environ. Sci. Technol., 2003, 37(9), 1744-1750.

81 C. H. Swartz, et al., Steroid estrogens, nonylphenol ethoxylate metabolites, and other wastewater contaminants in groundwater affected by a residential septic system on Cape Cod, MA, Environ. Sci. Technol., 2006, 40(16), 48944902.

82 L. Ma, S. R. Yates and D. Ashworth, Parent and conjugated estrogens and progestagens in surface water of the Santa Ana River: Determination, occurrence, and risk assessment, Environ. Toxicol. Chem., 2016, 35(11), 2657-2664.

$83 \mathrm{X}$. Bai, et al., Dissipation and transformation of $17 \beta$-estradiol-17-sulfate in soil-water systems, J. Hazard. Mater., 2013, 260, 733-739.

$84 \mathrm{~W}$. Ben, et al., Transformation and fate of natural estrogens and their conjugates in wastewater treatment plants: Influence of operational parameters and removal pathways, Water Res., 2017, 124, 244-250. 